\title{
Complexity Reduced Multipath Mitigation in GNSS with the GRANADA Bit-True Software Receiver
}

\author{
Ingmar Groh, Stephan Sand, and Christian Mensing \\ German Aerospace Center (DLR), \\ Institute for Communications and Navigation, \\ 82234 Wessling, Germany, \\ E-mail \{Ingmar.Groh, Stephan.Sand, Christian.Mensing\}@dlr.de.
}

\begin{abstract}
The positioning performance of global navigation satellite systems (GNSSs) mass market receivers severely degrades when the received satellite signals are subject to multipath propagation. Therefore, the estimation of several unknown channel amplitudes and taps in a multipath environment is an important approach to mitigate the multipath effects. In professional receivers, viable multipath mitigation approaches are the maximum likelihood (ML) estimator, the expectation maximization (EM) approach and the space alternating generalized expectation maximization (SAGE) algorithm. However, all methods require a high computational complexity when used in spread spectrum systems due to long spreading sequences. Therefore, one contribution of this paper is that we apply subspace methods to decrease the computational complexity before executing the above iterative estimation algorithms. Further, we assess respective performance of the algorithms and the future Galileo navigation system by using the Galileo receiver analysis and design application (GRANADA) simulator. The complexity reduction algorithms are specifically adjusted to the E1 Galileo binary offset carrier (BOC) signal, which superimposes data and pilot signals. Moreover, we adapt the complexity reduction so that it can handle any sampling frequency that is not necessarily an integer multiple of the chip rate.

Index Terms - spread spectrum system, synchronization, maximum likelihood channel estimation, EM and SAGE algorithm, GRANADA simulator, complexity reduction
\end{abstract}

\section{INTRODUCTION}

Position estimation in global navigation satellite systems (GNSSs) like the GALILEO system [1] is typically performed using pseudorange measurements between several satellites and the receiver. A precise location determination requires the synchronization of the respective line of sight (LOS) path between the transmitters and the receivers with sub chip accuracy. In order to test the synchronization performance of GALILEO navigation receivers, the GRANADA (Galileo Receiver $\underline{\text { ANalysis }}$ And $\underline{\text { Design }}$ Application) simulator [2], [3] has been developed. In a multipath propagation environment, the reception of signal replicas results in biased pseudoranges that will deteriorate the position estimation. Hence, it is necessary to mitigate the multipath effects in an efficient way. In a mass market receiver, this may be partially achieved by a narrow early minus late (NEML) or other correlators [4].

Alternative to the correlator approach, maximum likelihood (ML) based multipath estimation nearly mitigates all adverse multipath effects on the pseudoranges [5]. A suboptimum multipath estimation based on the iterative expectation maximization (EM) algorithm [6] or the improved space alternating generalized expectation maximization (SAGE) algorithm [7] has been investigated by [8]. J. Selva developed in [9], [10], [11] the framework for complexity reduced maximum likelihood (ML) channel estimation (CE) methods in navigation receivers which allows an efficient implementation. This framework comprised different subspace approaches which consisted of code or signal matched filter (MF) banks or principal components (PCs) filter or a suitable combination of these two algorithms.

However, efficient multipath mitigation algorithms have not yet been integrated into the GRANADA simulation tool and evaluated. In this paper, we combine the complexity reduction together with the EM, ML, and SAGE algorithms. We implement and investigate the performance of these algorithms in the GRANADA simulation tool. Specifically, we analyze the performance for a Galileo E1 $\mathrm{BOC}(1,1)$ signal which is comprised of the superposition of pilot and data symbols Further, we adapt the complexity reduction so that it works also for non integer oversampling factors.

Notation: Vectors, e.g. $\boldsymbol{y} \in \mathbb{C}^{N}$, and matrices, e.g. $\boldsymbol{A} \in$ $\mathbb{C}^{\overline{N \times M}}$, are denoted by small and captial bold letters. Random quantities (e.g. $\boldsymbol{y} \in \mathbb{C}^{N}$ ) are distinguised from deterministic

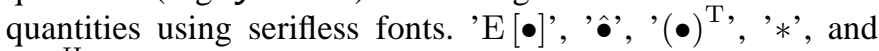
' $(\bullet)^{\mathrm{H}}$, denote the expectation, estimation, transposed, complex conjugate, and Hermitian operators. The operator $\operatorname{diag}\{\boldsymbol{x}\} \in$ $\mathbb{C}^{N \times N}$ denotes the diagonal matrix with the elements of $\boldsymbol{x} \in$ $\mathbb{C}^{N}$ on its diagonal. $\boldsymbol{y}:=$ FFT $\{\boldsymbol{x}\} \in \mathbb{C}^{N}$ represents the fast Fourier transform (FFT) of a vector $\boldsymbol{x}$ whose dimension $N$ is a power of 2. $\mathbf{0}_{N} \in \mathbb{C}^{N}$ and $\mathbf{1}_{N} \in \mathbb{C}^{N \times N}$ represent the $N$-dimensional column vector with zero elements and the $N$ dimensional unit matrix, respectively.

\section{CHANNEL AND TRANSMISSION MODEL}

The GRANADA simulator models the propagation channel as a Ricean fading channel of length $L$ [12]

$$
\mathrm{h}(\tau, t):=\sum_{\ell=0}^{L-1} \mathrm{~h}_{\ell}(t) \delta\left(\tau-\tau_{\ell}\right)
$$

where $\mathrm{h}_{\ell}(t)$ denotes the timevariant channel coefficients, and $\delta\left(\tau-\tau_{\ell}\right)$ the Dirac function. The power delay profile of $\mathrm{h}(\tau, t)$ is

$$
\mathrm{E}\left[|\mathrm{h}(\tau, t)|^{2}\right]=\sum_{\ell=0}^{L-1} a_{\ell}^{2} \delta\left(\tau-\tau_{\ell}\right)
$$


with $\mathrm{E}\left[\left|\mathrm{h}_{\ell}(t)\right|^{2}\right]=a_{\ell}^{2}$. We have for $\ell=1, \ldots, L-1$

$$
\mathrm{h}_{\ell}(t) \sim \mathcal{N}_{\mathrm{c}}\left(0, a_{\ell}^{2}\right)
$$

and at $\ell=0$

$$
\mathrm{h}_{0}(t) \sim \mathcal{N}_{\mathrm{c}}\left(1, a_{0}^{2}\right) .
$$

The power delay profile also defines the Ricean factor

$$
K_{\mathrm{RICEAN}}=-10 \log _{10}\left(\sum_{\ell=0}^{L-1} a_{\ell}^{2}\right) .
$$

We observe the transition to an $L$ path Rayleigh fading channel if $K_{\text {RICEAN }} \rightarrow-\infty$, and to an additive white Gaussian noise (AWGN) channel model if $K_{\text {RICEAN }} \rightarrow \infty$.

The channel described in Eqn. (1) represents the standard GRANADA channel implementation. The Doppler bandwidth values however are in the ranges of several $\mathrm{Hz}$ only, i.e. the random channel amplitude functions $c_{0}(t), \ldots, c_{L-1}(t)$ can be approximated by $a_{0}, \ldots, a_{L-1}$. For the algorithmic adaptation of the multipath mitigation presented in the following section to timevariant channels, we refer to [9]. By means of these assumptions, we obtain the Rx signal vector

$$
\boldsymbol{y}:=\sum_{\ell=0}^{L-1} a_{\ell} \boldsymbol{s}\left(\tau_{\ell}\right)+\boldsymbol{n}=\boldsymbol{S}(\boldsymbol{\tau}) \boldsymbol{a}+\boldsymbol{n} \in \mathbb{C}^{M N Q},
$$

where $\boldsymbol{n} \sim \mathcal{N}_{\mathrm{c}}\left(\mathbf{0}_{M N Q}, \sigma_{n}^{2} \mathbf{1}_{M N Q}\right)$ describes the zero mean additive white Gaussian noise (AWGN) vector, $\boldsymbol{S}(\boldsymbol{\tau})=$ $\left[\boldsymbol{s}\left(\tau_{0}\right), \ldots, \boldsymbol{s}\left(\tau_{L-1}\right)\right] \in \mathbb{C}^{M N Q \times L}$ the signal matrix, and $\boldsymbol{a}=$ $\left[a_{0}, \ldots, a_{L-1}\right]^{\mathrm{T}} \in \mathbb{C}^{L}$ forms the amplitude vector. The SNR $\gamma$ is defined according to Eqn. (6) as $\gamma:=\left(\sum_{\ell=0}^{L-1}\left|a_{\ell}\right|^{2}\right) / \sigma_{n}^{2}$. $M$ successive observation and noise vectors $\boldsymbol{y}_{m}, \boldsymbol{n}_{m} \in \mathbb{C}^{N Q}$ $(m=1, \ldots, M)$ form the whole observation and vector $\boldsymbol{y}=\left[\boldsymbol{y}_{1}^{\mathrm{T}}, \ldots, \boldsymbol{y}_{M}^{\mathrm{T}}\right]^{\mathrm{T}}, \boldsymbol{n}=\left[\boldsymbol{n}_{1}^{\mathrm{T}}, \ldots, \boldsymbol{n}_{M}^{\mathrm{T}}\right]^{\mathrm{T}} \in \mathbb{C}^{M N Q}$.

\section{INTERPOLATION MODEL}

In this section, we analyze the generation of the baseband transmit (Tx) signal in GRANADA [1]. The Tx signal in the E1 band comprises several parts of which only the data and pilot signals are relevant for a mass market receiver and hence, for multipath mitigation. The application of the algorithmic multipath mitigation requires an efficient mathematical description for the generation of time delayed direct sequence spread spectrum (DS-SS) signals, which is given in the next paragraphs.

As a pulse shape for the transmission considered in this paper, GRANADA uses binary offset carrier (BOC) pulses $g(t)$ which are defined by the settings for the Tx and the receive (Rx) filter, in particular by the one sided limit frequency $f_{\mathrm{N}}$ of the Rx filter. We may write the DS-SS Tx signal as

$$
\begin{aligned}
\mathrm{s}(t) & :=\sum_{m=0}^{M-1} d_{\mathrm{P}, m} \sum_{n=0}^{N-1} c_{\mathrm{P}, n} \mathrm{~g}\left(t-m T-n T_{\mathrm{C}}\right), \\
& +\sum_{m=0}^{M-1} d_{\mathrm{D}, m} \sum_{n=0}^{N-1} c_{\mathrm{D}, n} \mathrm{~g}\left(t-m T-n T_{\mathrm{C}}\right)
\end{aligned}
$$

where $T$ and $T_{\mathrm{C}}=1 / R_{\mathrm{C}}$ denote the frame duration and chip duration with $R_{\mathrm{C}}$ being the chip rate. The pilot and data symbol sequences $d_{\mathrm{P}, m}$ and $d_{\mathrm{D}, m}, m=0, \ldots, M-1$ are taken from binary phase shift keying (BPSK) modulation.The spreading code sequences $c_{\mathrm{P}, n}$ for the pilots and $c_{\mathrm{D}, n}, \quad(n=$ $0, \ldots, N-1)$ for the data are orthogonal Galileo codes of length $N$. If we assume an observation interval of duration $M N T_{\mathrm{C}}$, we can define $M$ successive signal vectors

$$
\boldsymbol{s}_{m}:=[\mathrm{s}[(m-1) N Q], \ldots, \mathrm{s}[m N Q-1]]^{\mathrm{T}} \in \mathbb{C}^{N Q},
$$

with $\mathrm{s}[k]:=\mathrm{s}\left(k / f_{\mathrm{S}}\right), k=(m-1) N Q, \ldots, m N Q-1$. For any sampling frequency $f_{\mathrm{S}}$ and a fixed chip rate $R_{\mathrm{C}}$, we have in mean $Q:=f_{\mathrm{S}} / R_{\mathrm{C}}$ samples per chip. Since $Q$ does not necessarily have to be an integer value, the samples in $\boldsymbol{s}_{m}$ are not uniformly distributed over the successive chips. This circumstance enforces a different kind of spreading signal interpolation for BOC pulses which is based on a decomposition of the BOC pulse into two rectangular (RECT) pulse halfs. The sampled Tx signal in matrix vector notation in Eqn. (7) states $\boldsymbol{s}=\left[\boldsymbol{s}_{1}^{\mathrm{T}}, \ldots, \boldsymbol{s}_{M}^{\mathrm{T}}\right]^{\mathrm{T}} \in \mathbb{C}^{M N Q}$, and

$$
\boldsymbol{s}_{m} \approx d_{\mathrm{P}, m} \boldsymbol{C}_{\mathrm{P}} \boldsymbol{g}+d_{\mathrm{D}, m} \boldsymbol{C}_{\mathrm{D}} \boldsymbol{g} .
$$

$\boldsymbol{C}_{\mathrm{P}}, \boldsymbol{C}_{\mathrm{D}} \in \mathbb{C}^{N Q \times N_{\mathrm{PP}}}$ are the pilot and data code convolution matrices which incorporate both the spreading and the BOC code, and $\boldsymbol{g} \in \mathbb{C}^{N_{\mathrm{PP}}}$ is the zero padded sampled and filtered RECT pulse which represents one half of BOC pulse. Even if $Q \in \mathbb{N}$, the sample assignment in GRANADA may not be uniform over all chips in the spreading signal. Hence, the sample assignment of the GRANADA Tx signal has to define the exact distribution of the pilot and data code values in $C_{\mathrm{P}}$ and $C_{\mathrm{D}}$ in order to construct an optimum signal interpolation for channel estimation algorithms that suffice navigation accuracy. Clearly, $\boldsymbol{g}$ incorporates both the Tx and Rx filter with their respective bandwidths. $N_{\mathrm{PP}}$ is chosen as the next power of two exceeding or equal to the number of pulse samples $N_{\mathrm{P}}$, for the FFT based pulse interpolation. Therefore, $\boldsymbol{g}$ contains the $N_{\mathrm{P}}$ samples of the RECT pulse padded two times with $N_{\mathrm{Z}}=\left(N_{\mathrm{PP}}-N_{\mathrm{P}}\right) / 2$ zeros, i. e.,

$$
\begin{aligned}
\boldsymbol{g}=[ & {\left[\mathbf{0}_{N_{\mathrm{Z}}}^{\mathrm{T}}, \mathrm{g}\left(-\left(N_{\mathrm{P}}-1\right) /\left(2 f_{\mathrm{S}}\right)\right), \ldots,\right.} \\
& \left.\mathrm{g}\left(\left(N_{\mathrm{P}}-1\right) /\left(2 f_{\mathrm{S}}\right)\right), \mathbf{0}_{N_{\mathrm{Z}}}^{\mathrm{T}}\right]^{\mathrm{T}} .
\end{aligned}
$$

The pulse interpolation technique enables the following pulse shifting [10]

$$
\mathbf{g}(\tau) \approx \boldsymbol{F}^{-1} \operatorname{diag}\{\operatorname{FFT}\{\boldsymbol{g}\}\} \boldsymbol{\psi}(\tau) .
$$

The matrix $\boldsymbol{F}^{-1} \in \mathbb{C}^{N_{\mathrm{PP}} \times N_{\mathrm{PP}}}$ is the permuted inverse Fourier matrix with elements

$$
\left(\boldsymbol{F}^{-1}\right)_{k \ell}=\exp \left(\mathrm{j}\left(2 \pi / N_{\mathrm{PP}}\right)\left(k-N_{\mathrm{PP}} / 2\right)\left(\ell-N_{\mathrm{PP}} / 2\right)\right),
$$

$k, \ell=1, \ldots, N_{\mathrm{PP}} . \psi(\tau) \in \mathbb{C}^{N_{\mathrm{PP}}}$ is a Vandermonde vector consisting of

$$
\begin{aligned}
& \quad(\psi(\tau))_{k}=\exp \left(-\mathrm{j}\left(2 \pi Q /\left(2 N_{\mathrm{PP}}\right)\right)\left(k-N_{\mathrm{PP}} / 2\right) \tau\right), \\
& k=1, \ldots, N_{\mathrm{PP}}
\end{aligned}
$$


Having determined the pulse interpolation in Eqn. (11), we can now formulate the interpolation representation of the spreading signal using the pilot and data code matrices $\boldsymbol{C}_{\mathrm{P}}, \boldsymbol{C}_{\mathrm{D}} \in \mathbb{C}^{N Q \times N_{\mathrm{PP}}}$ as $\mathbf{s}(\tau)=\left[\mathbf{s}_{1}^{\mathrm{T}}(\tau), \ldots, \mathbf{s}_{M}^{\mathrm{T}}(\tau)\right]^{\mathrm{T}} \in$ $\mathbb{C}^{M N Q}$, where

$$
\begin{aligned}
\mathbf{s}_{m}(\tau) & =d_{\mathrm{P}, m} \boldsymbol{C}_{\mathrm{P}} \mathbf{g}(\tau)+d_{\mathrm{D}, m} \boldsymbol{C}_{\mathrm{D}} \mathbf{g}(\tau) \\
& =d_{\mathrm{P}, m} \mathbf{s}_{\mathrm{P}}(\tau)+d_{\mathrm{D}, m} \mathbf{s}_{\mathrm{D}}(\tau)
\end{aligned}
$$

for $m=1, \ldots, M$. The abbreviations $C_{\mathrm{P}} \mathbf{g}(\tau)=\mathbf{s}_{\mathrm{P}}(\tau)$ and $C_{\mathrm{D}} \mathbf{g}(\tau)=\mathrm{s}_{\mathrm{D}}(\tau)$ represent the interpolated pilot and data signal.

At this point it is convenient to derive a second interpolation representation for the pilot and data code $c_{P}, c_{D} \in \mathbb{C}^{N}$. The code interpolation is based on the same interpolation method as Eqn. (11). Again, the two distinct parameters are $f_{\mathrm{N}}$ as band limiting and frequency $f_{\mathrm{S}}$ as sampling frequency of the BOC pulse. A Dirac delta function $\delta(t)$ which is transformed into the frequency domain, bandlimited to $f_{\mathrm{N}}$, and transformed back into time domain yields the sinc function

$$
\delta(t) \approx \operatorname{sinc}(t)=\sin \left(2 \pi f_{\mathrm{N}} t\right) /(\pi t) .
$$

We proceed now in the same way as before with the interpolation of the RECT pulse: $\delta \in \mathbb{C}^{N_{\mathrm{PP}}}$ contains the $N_{\mathrm{P}}$ samples of the sinc function padded two times with $N_{\mathrm{Z}}=\left(N_{\mathrm{PP}}-N_{\mathrm{P}}\right) / 2$ zeros to reach $N_{\mathrm{PP}}$, i. e.

$$
\begin{gathered}
\boldsymbol{\delta}=\left[\mathbf{0}_{N_{\mathrm{Z}}}^{\mathrm{T}}, \delta\left(-\left(N_{\mathrm{P}}-1\right) /\left(2 f_{\mathrm{S}}\right)\right), \ldots,\right. \\
\left.\delta\left(\left(N_{\mathrm{P}}-1\right) /\left(2 f_{\mathrm{S}}\right)\right), \mathbf{0}_{N_{\mathrm{Z}}}^{\mathrm{T}}\right]^{\mathrm{T}} .
\end{gathered}
$$

The corresponding interpolation representation to Eqn. (11) is then

$$
\boldsymbol{\delta}(\tau) \approx \boldsymbol{F}^{-1} \operatorname{diag}\{\mathrm{FFT}\{\boldsymbol{\delta}\}\} \boldsymbol{\psi}(\tau) .
$$

Similar to Eqn. (11), we define as interpolated pilot code $\mathbf{c}_{\mathrm{P}}(\tau)=\boldsymbol{C}_{\mathrm{P}} \boldsymbol{\delta}(\tau)$ and $\mathbf{c}_{\mathrm{D}}(\tau)=\boldsymbol{C}_{\mathrm{D}} \boldsymbol{\delta}(\tau)$ as interpolated data code.

\section{Channel Estimation in Reduced Dimension}

For $\boldsymbol{y} \in \mathbb{C}^{M N Q}$, the ML estimate $\{\hat{\boldsymbol{a}}, \hat{\boldsymbol{\tau}}\} \in \mathbb{C}^{L}$ is found according to

$$
\{\hat{\boldsymbol{a}}, \hat{\boldsymbol{\tau}}\}=\underset{\{\boldsymbol{a}, \boldsymbol{\tau}\}}{\operatorname{argmin}}\|\boldsymbol{y}-\mathbf{S}(\boldsymbol{\tau}) \boldsymbol{a}\|_{2}^{2} .
$$

To obtain a low complexity solution, we propose to use the following two stage complexity reduction.

As a first step, the pilot symbols in the sampled Rx vector $\boldsymbol{y}$ are demodulated and filtered codeword by codeword by an orthonormalized correlatorbank of $N_{\mathrm{CC}}$ pilot code matched correlators (CMCs) $\boldsymbol{Q}_{\mathrm{CC}} \in \mathbb{C}^{N Q \times N_{\mathrm{CC}}}$.

This matrix $Q_{\mathrm{CC}} \in \mathbb{C}^{N Q \times N_{\mathrm{CC}}}$ is obtained from a $\mathrm{QR}$ decomposition of $\boldsymbol{C}_{\mathrm{CC}}=\left[\mathbf{c}_{\mathrm{P}}\left(\tau_{\mathrm{G}, 1}\right), \ldots, \mathbf{c}_{\mathrm{P}}\left(\tau_{\mathrm{G}, N_{\mathrm{CC}}}\right)\right]=\boldsymbol{Q}_{\mathrm{CC}} \boldsymbol{R}_{\mathrm{CC}}$ yielding the output of the correlatorbank as

$$
\begin{aligned}
\boldsymbol{y}_{\mathrm{CC}} & :=\sum_{m=1}^{M} d_{\mathrm{P}, m}^{*} \boldsymbol{Q}_{\mathrm{CC}}^{\mathrm{H}} \boldsymbol{y}_{m}=\sum_{m=1}^{M} d_{\mathrm{P}, m}^{*} \boldsymbol{Q}_{\mathrm{CC}}^{\mathrm{H}}\left(\sum_{\ell=0}^{L-1} a_{\ell} \mathbf{s}_{m}\left(\tau_{\ell}\right)+\boldsymbol{n}_{m}\right) \\
& =M \boldsymbol{Q}_{\mathrm{CC}}^{\mathrm{H}} \sum_{\ell=0}^{L-1} a_{\ell} \mathbf{S}_{\mathrm{P}}\left(\tau_{\ell}\right)+\sum_{m=1}^{M} d_{\mathrm{P}, m}^{*} \boldsymbol{Q}_{\mathrm{CC}}^{\mathrm{H}} \boldsymbol{n}_{m} \in \mathbb{C}^{N_{\mathrm{CC}}}, \quad(19)
\end{aligned}
$$

where we have exploited the orthogonality between the pilot CMC matrix $\boldsymbol{Q}_{\mathrm{CC}} \in \mathbb{C}^{N Q \times N_{\mathrm{CC}}}$ and the data code matrix $\boldsymbol{C}_{\mathrm{D}} \in \mathbb{C}^{N Q \times N_{\mathrm{CC}}} \cdot \boldsymbol{\tau}_{\mathrm{G}}=\left[\tau_{\mathrm{G}, 1}, \ldots, \tau_{\mathrm{G}, N_{\mathrm{CC}}}\right]^{\mathrm{T}} \in \mathbb{C}^{N_{\mathrm{CC}}}$ defines the respective positions of the $N_{\mathrm{CC}} \mathrm{CMCs}$. The index represents the abbreviation canonical components (CCs), which is an alternative expression for signal compression using matched filter banks [9]. In the following further complexity reduction using principal components (PCs), the introduction of the filtered pilot signal $\mathrm{s}_{\mathrm{P}, \mathrm{CC}}(\tau)=Q_{\mathrm{CC}}^{\mathrm{H}} \mathbf{s}_{\mathrm{P}}(\tau) \in \mathbb{C}^{N_{\mathrm{CC}}}$ is helpful. We calculate the autocorrelation matrices

$$
\boldsymbol{R}_{\mathrm{SP}_{\mathrm{P}}(\boldsymbol{\tau})}=\mathrm{E}\left[\mathbf{s}_{\mathrm{P}}(\boldsymbol{\tau}) \mathbf{S}_{\mathrm{P}}(\tau)^{\mathrm{H}}\right]
$$

and

$$
\boldsymbol{R}_{\mathrm{S}_{\mathrm{P}, \mathrm{CC}}(\boldsymbol{\tau})}=\mathrm{E}\left[\mathrm{s}_{\mathrm{P}, \mathrm{CC}}(\boldsymbol{\tau}) \mathbf{s}_{\mathrm{P}, \mathrm{CC}}(\boldsymbol{\tau})^{\mathrm{H}}\right]=\boldsymbol{Q}_{\mathrm{CC}}^{\mathrm{H}} \boldsymbol{R}_{\mathrm{SP}_{\mathrm{P}}(\boldsymbol{\tau})} \boldsymbol{Q}_{\mathrm{CC}}
$$

exploiting a-priori information about the channel taps. Here, we choose a robust a-priori assumption that is a uniform distribution in $\left[-\tau_{\mathrm{MAX}}, \tau_{\mathrm{MAX}}\right]$ [10], [11] (in general, we set $\tau_{\mathrm{MAX}}=T_{\mathrm{C}}$ which yields the tracking range for fine synchronization):

$$
\boldsymbol{R}_{\mathrm{SP}_{\mathrm{P}}(\boldsymbol{\tau})}=\mathrm{E}\left[\mathbf{s}_{\mathrm{P}}(\boldsymbol{\tau}) \mathbf{s}_{\mathrm{P}}(\boldsymbol{\tau})^{\mathrm{H}}\right]=\int_{-\tau_{\mathrm{MAX}}}^{\tau_{\mathrm{MAX}}} \mathbf{s}_{\mathrm{P}}(\boldsymbol{\tau}) \mathbf{s}_{\mathrm{P}}(\boldsymbol{\tau})^{\mathrm{H}} \frac{1}{2 \tau_{\mathrm{MAX}}} \mathrm{d} \tau
$$

For the second stage of complexity reduction, it is necessary to calculate the eigenvalue decomposition (EVD) of

$$
\boldsymbol{R}_{\mathrm{s}, \mathrm{CC}}(\boldsymbol{\tau})=\boldsymbol{Q} \Lambda \boldsymbol{Q}^{\mathrm{H}}
$$

$\boldsymbol{Q}:=\left[\boldsymbol{q}_{1}, \ldots, \boldsymbol{q}_{N_{\mathrm{CC}}}\right] \in \mathbb{C}^{N_{\mathrm{CC}} \times N_{\mathrm{CC}}}$ contains in its columns the respective eigenvectors and $\boldsymbol{\Lambda}:=\operatorname{diag}\left\{\left[\lambda_{1}, \ldots, \lambda_{N_{\mathrm{CC}}}\right]^{\mathrm{T}}\right\} \in$ $\mathbb{C}^{N_{\mathrm{CC}} \times N_{\mathrm{CC}}}$ is the diagonal matrix containing the eigenvalues sorted in descending order, i.e. $\lambda_{1}>\cdots>\lambda_{N_{\mathrm{CC}}}>0$. The use of a given number $N_{\mathrm{PC}} \leq N_{\mathrm{CC}}$ of PCs yields the approximation

$$
\boldsymbol{R}_{\mathrm{s}, \mathrm{CC}}(\boldsymbol{\tau})=\sum_{k=1}^{N_{\mathrm{PC}}} \lambda_{k} \boldsymbol{q}_{k} \boldsymbol{q}_{k}^{\mathrm{H}} .
$$

As a result, we have

$$
\boldsymbol{y}_{\mathrm{PC}}:=\boldsymbol{Q}_{\mathrm{PC}}^{\mathrm{H}} \boldsymbol{y}_{\mathrm{CC}} \in \mathbb{C}^{N_{\mathrm{PC}}},
$$

where $\boldsymbol{Q}_{\mathrm{PC}}:=\left[\boldsymbol{q}_{1}, \ldots, \boldsymbol{q}_{N_{\mathrm{PC}}}\right] \in \mathbb{C}^{N_{\mathrm{CC}} \times N_{\mathrm{PC}}}$ is the PC eigenspace matrix. At this place it is also convenient to define the twofold filtered pilot signal $\mathbf{s}_{\mathrm{P}, \mathrm{PC}}(\tau)=\boldsymbol{Q}_{\mathrm{PC}}^{\mathrm{H}} \boldsymbol{Q}_{\mathrm{CC}}^{\mathrm{H}} \mathbf{s}_{\mathrm{P}}(\tau) \in$ $\mathbb{C}^{N_{\text {PC }}}$. After this two stage complexity reduction, we apply three different methods for determining the solution of the ML estimation problem. The first algorithm which we term complexity reduced multipath mitigation (CRMM) combines a least squares (LS) estimation for the channel amplitudes with a multidimensional Newton algorithm for minimizing the resulting ML cost function [9]. The two other approaches, the expectation maximization (EM) algorithm [6] as well as the space alternating generalized expectation maximization (SAGE) method [7], resort to a componentwise LS amplitude estimation and a correlation maximization to determine the channel delays. 


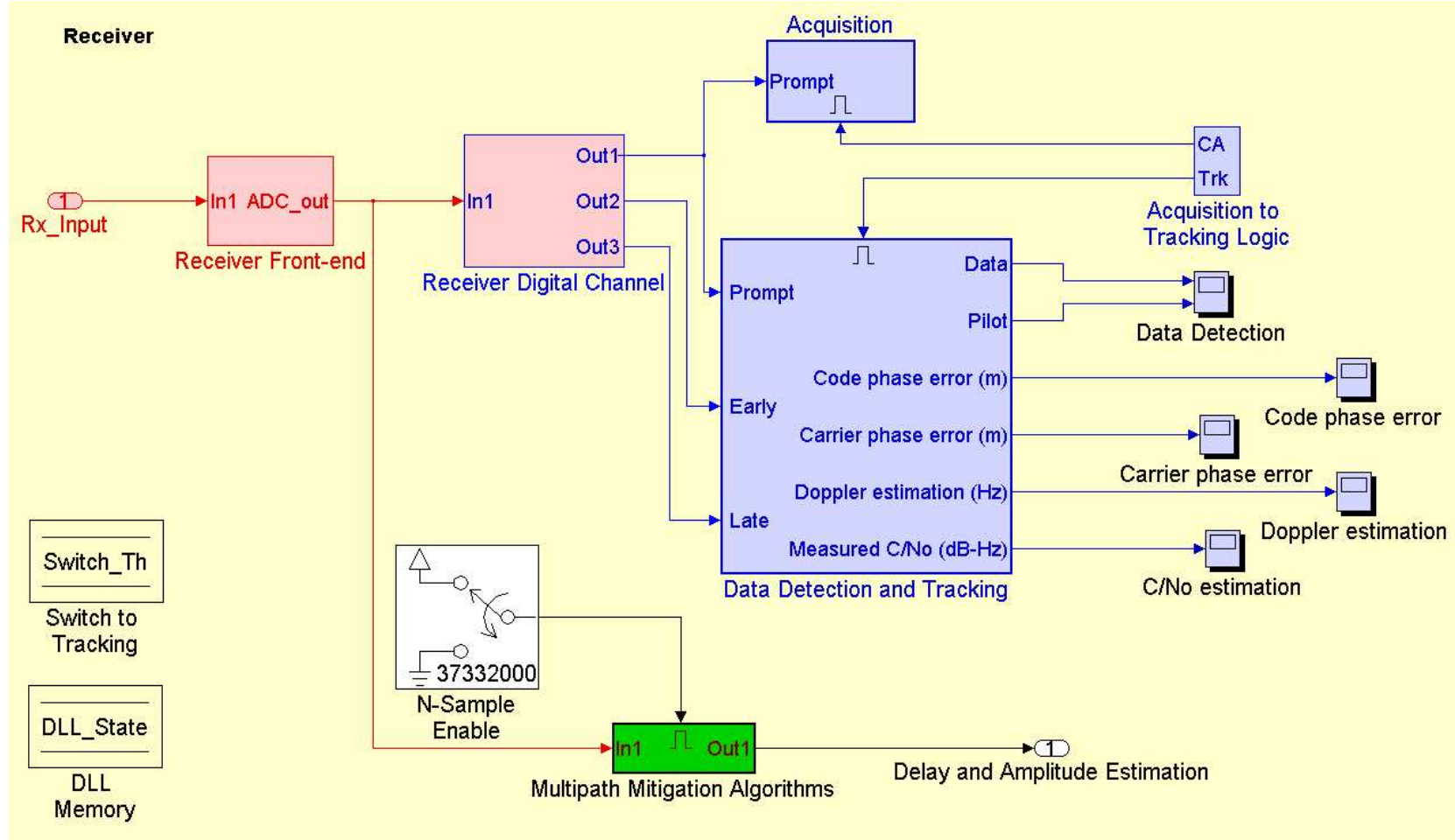

Figure 1. Receiver in GRANADA

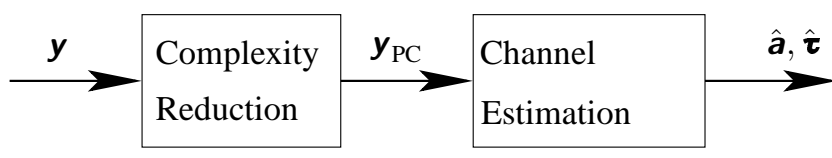

Figure 2. Multipath Mitigation in GRANADA

Table I

List OF USED Simulation PARAMETERS

\begin{tabular}{|r|l|}
\hline parameter & value \\
\hline one sided limit frequency (BOC pulse): & $f_{\mathrm{N}}=40.92 \mathrm{MHz}$ \\
physical sampling frequency: & $f_{\mathrm{S}}=93.33 \mathrm{MHz}$ \\
chip rate: & $R_{\mathrm{C}}=1.023 \mathrm{MHz}$ \\
code length (Galileo code): & 4092 \\
observation time: & $200 \mathrm{~ms}(M=50)$ \\
\hline
\end{tabular}

\section{A. GRANADA Implementation}

In the following, we present the GRANADA implementation of the above complexity reduced channel estimation in Figs. 1 and 2.

Fig. 1 shows the place of the integration of the multipath estimation for non synchronized signals. Once the time instant $0.4 \mathrm{~s}$ is exceeded, the block for the complexity reduced multipath mitigation is enabled, and all the successive signal samples are processed by the estimation algorithms. The time offset is necessary due to the transient duration of $0.4 \mathrm{~s}$ of the code tracking loops (to be found in the block data detection and tracking), cf. [1], [2].

Fig. 2 depicts the complexity reduced multipath mitigation in more detail. The received vector $\boldsymbol{y}$ is first complexity reduced according to Eqns. (19) and (25). For instance, for the implementation in Sec. V, the input vector $\boldsymbol{y}$ is reduced from 373320 samples to the output vector of the complexity reduction $\boldsymbol{y}_{\mathrm{PC}} 20$. After that, we apply the channel estimation algorithms, e.g. the ML, EM, or SAGE.

\section{Simulations And Results}

Tab. I summarizes the parameters for the following two example simulations of complexity reduced multipath mitigation algorithms in the Galileo simulator GRANADA. Fig. 3 depicts the bias result of the presented complexity reduced estimation algorithms when considering a two path $(L=2)$ channel model with fixed amplitude functions $\mathrm{h}_{0}(t)=0$ and $\mathrm{h}_{1}(t)= \pm 1 / \sqrt{2}$. The complexity reduction comprises both a CC analysis (CCA) and a successive PC analysis (PCA). We observe that the positioning error for a narrow correlator [5] is about $12 \mathrm{~m}$ maximum, and for the narrow correlator tracking structure, the multipath bias decreases with increasing $\tau_{1}$. This holds both for the inphase and outofphase channel constellation. The inphase and outofphase channel constellation yield bias values which are approximately equal in magnitude with different sign throughout the considered direct multipath delay difference range. Contrary, the complexity reduced estimators with two degrees of freedom allow a bias free position estimation. The slight errors which these 


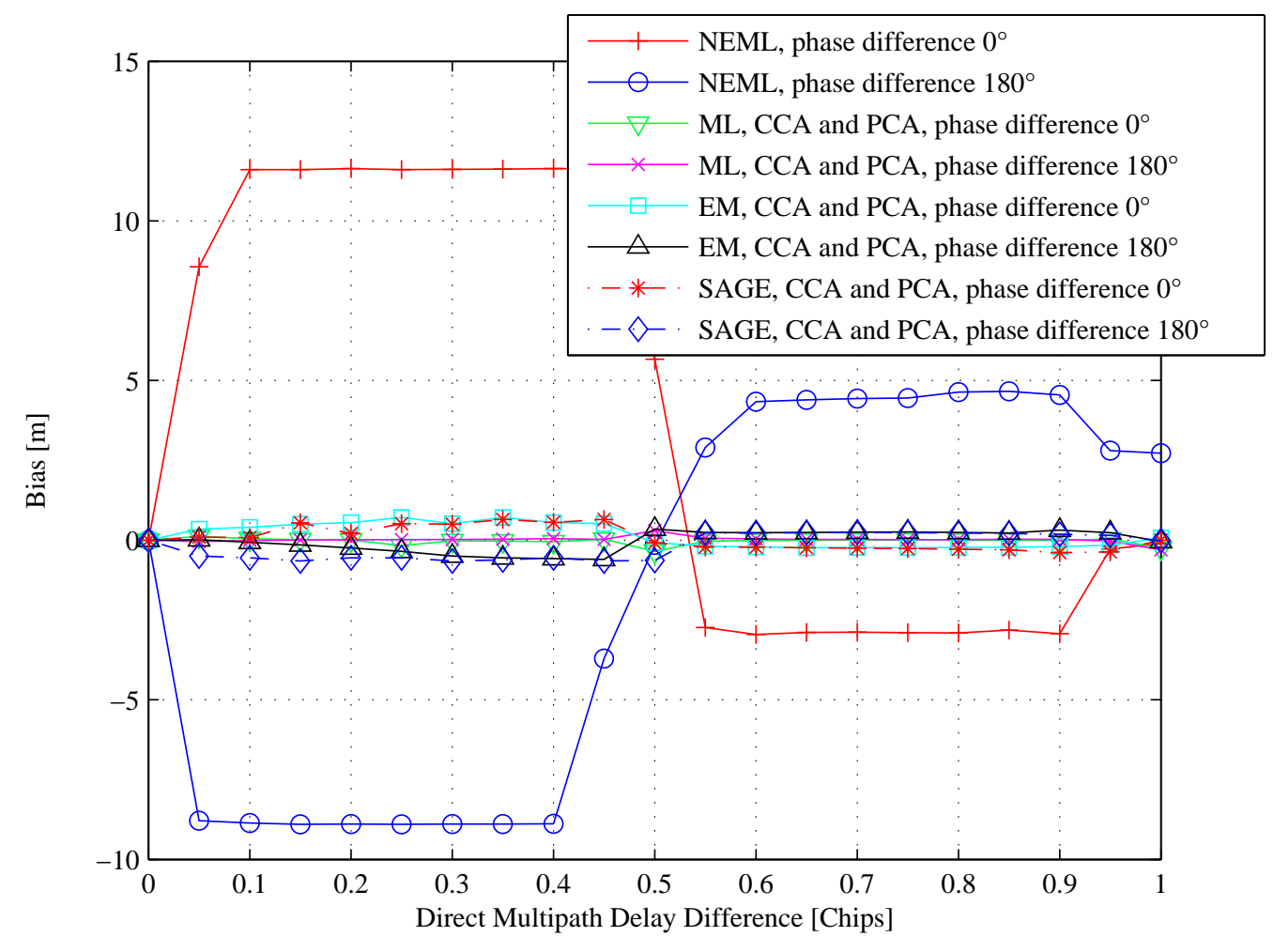

Figure 3. Bias [m] versus the Direct Multipath Delay Difference for a Fixed Channel of Length $L=2$

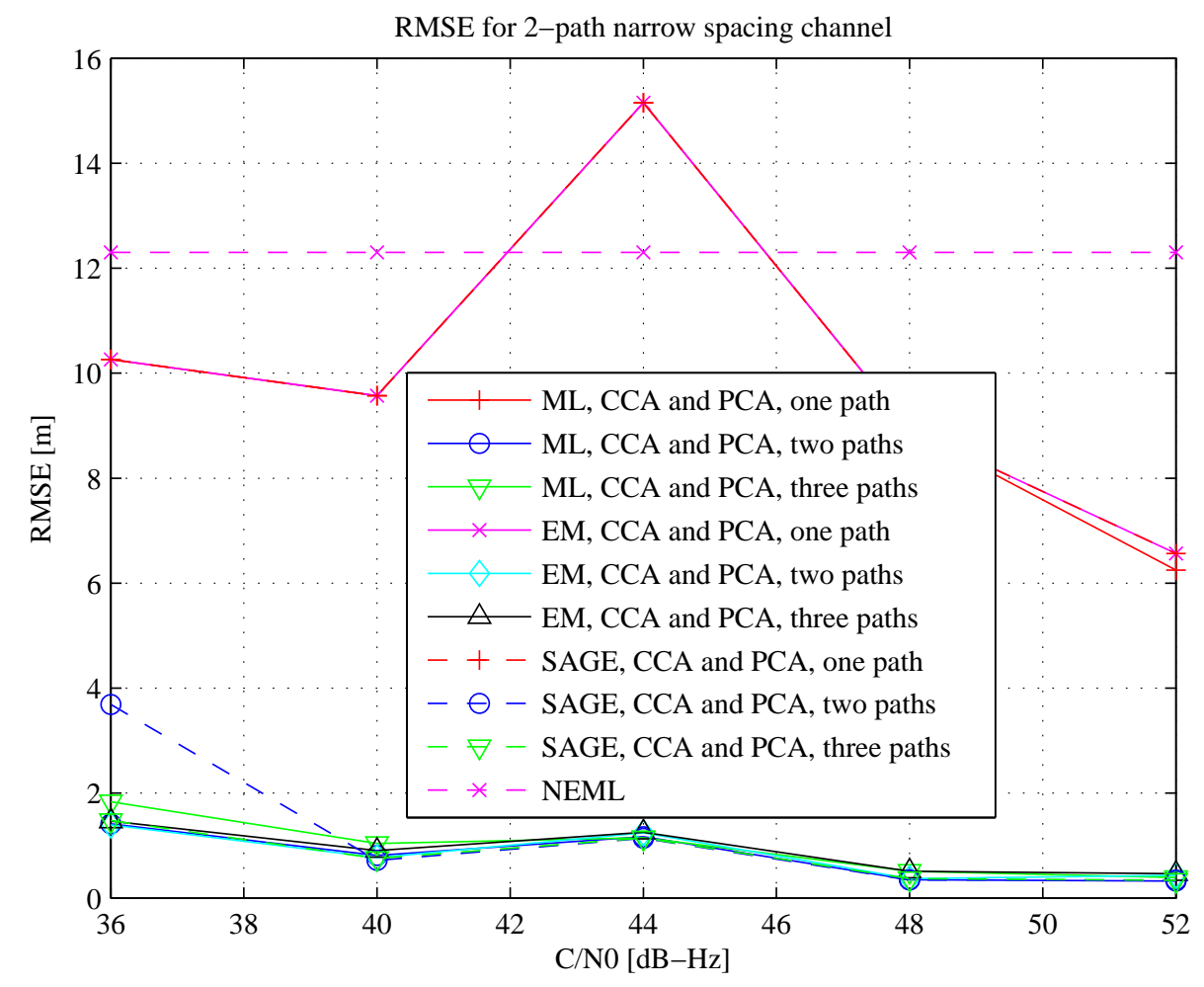

Figure 4. RMSE [m] versus the Carrier to Noise Ratio [dB-Hz] for a Two Path Narrow Spacing Channel 
implementations still have are due to the signal interpolation approximations in Sec. III. This interpolation error is however substantially smaller than the magnitude of the bias resulting from the narrow correlator. Fig. 3 proves therefore the ability of low complexity multipath estimators to remove a multipath position bias which the narrow correlator tracking structure cannot prevent.

Next, the behaviour of the multidimensional estimation algorithms are analyzed with respect to their noise performance. Fig. 4 shows the root mean square error (RMSE) result of different complexity reduced estimation algorithms after application to a two path $(L=2)$ narrow spacing $\left(\tau_{0} / T_{\mathrm{C}}=0\right.$, $\left.\tau_{1} / T_{\mathrm{C}}=0.1\right)$ Ricean channel $\left(a_{0}=1 / \sqrt{10}, a_{1}=1 / \sqrt{2}\right)$. The channel dynamics have a $4 \mathrm{~Hz}$ Doppler bandwidth for all paths. The curves in Fig. 4 belonging to the estimators which have one degree of freedom only reveal a bias value of about $10 \mathrm{~m}$. This order of magnitude corresponds to the maximum bias in the previous simulation in Fig. 3 for the narrow correlator [4]. Concerning the two and three path estimators, we observe a RMSE between $2 \mathrm{~m}$ to $0.5 \mathrm{~m}$ which decreases continuously for increasing $C / N_{0}$ and which is only due to the noise and the interpolation error. It is also important to mention that despite the channels timevariance of $4 \mathrm{~Hz}$, the approximation in the derivation in Secs. II and IV is justified. The small RMSE for the two and three dimensional estimators confirms the applicability of this simplification. Another important observation from Fig. 4 is the robustness with respect to the model order selection. For any of the suggested complexity reduced multipath estimators, the estimation performance for two and three degrees of freedom are almost identical.

\section{CONCLUSIONS}

In this paper, we reviewed three low complexity solutions for the maximum likelihood (ML) estimation of several unknown channel amplitudes and taps in a multipath environment for the design of positioning receivers. These iterative algorithms are either based on a direct minimization of the cost function or on a separate solution for each dimension like the expectation maximization (EM) approach and the space alternating generalized expectation maximization (SAGE) algorithm. Since all three methods require a high computational complexity due to the long spreading sequences used in navigation systems, we have developed solutions for decreasing the computational complexity before executing the presented iterative estimation algorithms.
The comprehensive GRANADA ( $\underline{\text { Galileo }}$ Receiver ANalysis And Design Application) simulator has been adapted for efficient multipath estimation and mitigation algorithms. Therefore, we complemented the bit true navigation receiver simulations in GRANADA and solved thereby two important problems. At first, we adapted the algorithms for complexity reduction used for maximum likelihood (ML) channel estimation to the superposition of data and pilot spreading signals in GRANADA. The second difficulty arised when implementing and testing interpolation based estimators, in particular for non-integer oversampling factors. We attacked this implementation task by modifying the signal and code interpolation according to the direct GRANADA signal generation.

\section{ACKNOWLEDGEMENT}

This work was partially carried out under GREAT (Galileo REceiver for mAss markeT) project, co-funded by the European GNSS Supervisory Authority (GSA) with funding from the 6th Framework Programme of the European Community for research and technological development.

\section{REFERENCES}

[1] GRANADA Interface Control Document, Issue 4.0, June 2005.

[2] GRANADA Software User Manual, Issue 2.0, June 2005.

[3] Galileo Open Service Signal In Space Interface Control Document (OS SIS ICD) Draft 0, May 2006.

[4] X. Hu and E. S. Lohan, "GRANADA Validation of Optimized Multiple Gate Delay Structures for Galileo SinBOC(1,1) Signal Tracking," in Proceedings of International Conference on ITS Telecommunications (ITST), Sophia Antipolis, France, June 2007.

[5] M. Lentmaier and B. Krach, "Maximum Likelihood Multipath Estimation in Comparison with Conventional Delay Locked Loops," in Proceedings of ION GNSS 19th International Technical Meeting of the Satellite Division (ION 2006), Fort Worth, Texas, September 2006.

[6] M. Feder and E. Weinstein, "Parameter Estimation of Superimposed Signals Using the EM Algorithm," IEEE Transactions on Acoustics, Speech, and Signal Processing, April 1988.

[7] B. Fleury, M. Tschudin, R. Heddergott, D. Dalhaus, and K. I. Pedersen, "Channel Parameter Estimation in Mobile Radio Environments using the SAGE Algorithm," IEEE Journal on Selected Areas in Communications, March 1999.

[8] F. Antreich, O. E. Rodríyguez, J. A. Nossek, and W. Utschick, "Estimation of Synchronization Parameters Using SAGE in a GNSS-Receiver," in Proceedings of ION GNSS 18th International Technical Meeting of the Satellite Division (ION 2005), Long Beach, California, September 2005.

[9] J. Selva, Efficient Multipath Mitigation Methods in Navigation Systems, Ph.D. thesis, Universitat Polytecnicia de Catalunya, Barcelona, Spain, 2004.

[10] J. Selva, "Complexity Reduction in the Parametric Estimation of Superimposed Signals," Signal Processing, vol. 84, pp. 2325-2343, 2004.

[11] J. Selva, "An Efficient Newton-Type Method for the Computation of ML Estimators in a Uniform Linear Array," IEEE Transactions on Signal Processing, vol. 53, no. 6, pp. 2036-2045, 2005.

[12] W. C. Jakes, Microwave Mobile Communications, John Wiley, IEEE Press, New York, USA, 1994. 